\title{
Correlation of Blood Lipid Profile, Blood Lipid Ratio and Cystatin C in Patients with Hyperthyroidism
}

\author{
Zhenjiang Hou ${ }^{1}$, Zhaoxin Mu ${ }^{1}$, Jingyu Zhang ${ }^{2}$ \\ Hong Fan ${ }^{2}$ and Jianzhang Hou ${ }^{3}$ \\ ${ }^{1}$ Institute of Thyroid Diseases, professor, Cangzhou Medical College, Cangzhou, China \\ ${ }^{2}$ Laboratory Diagnostics Division, Hebei Cangzhou Hospital of Integrated Traditional Chinese and \\ Western Medicine, Cangzhou, China \\ ${ }^{3}$ Department of surgery, Cangzhou people's hospital, Cangzhou medical college, Cangzhou, China
}

Keywords: Hyperthyroidism; Subclinical Hyperthyroidism; Blood Lipid Profile; Blood Lipid Ratios; cystatin C

\begin{abstract}
To investigate the correlation among serum lipids and their ratios and cystatin $\mathrm{C}$ (CysC) in patients with hyperthyroidism. authors respectively chose fifty patients of hyperthyroidism, subclinical hyperthyroidism and the control group. TC, TG and LDL-C in the hyperthyroidism and subclinical hyperthyroidism groups were significantly lower than those in the control group ( $\mathrm{P}$ $<0.01$ ); TC/HDL-C, TG/HDL-C, LDL-C/HDL-C, LCI, and non-HDL-C in the hyperthyroidism group were significantly higher than those in the subclinical hyperthyroidism and control groups; TC/HDL-C and TG/HDL-C in the subclinical hyperthyroidism group were significantly higher those in the control group while LDL-C/HDL-C, LCI, and non-HDL-C were significantly lower than the control group. LCI decrease being the most significant followed by LDL-C/HDL-C. The thyroid hormones mostly correlated with TC,followed by non-HDL-C, LDL-C and LCI. The level of $\mathrm{CysC}$ in the hyperthyroidism and subclinical hyperthyroidism groups was significantly higher and there was also significant difference regarding this level between both groups $(\mathrm{P}<0.05)$. The author came to the conclusion that the changes of serum lipid ratios in patients with hyperthyroidism and subclinical hyperthyroidism were more obvious than single indicator; and their CysC level was also significantly higher than that of control group. Dynamic monitoring of blood lipid ratios and $\mathrm{CysC}$ level could be an optimized combination item for the transformation from subclinical hyperthyroidism to hyperthyroidism and prediction of hyperthyroidism complicated with CHD.
\end{abstract}

\section{Introduction}

Hyperthyroidism is an endocrine disorder caused by abnormal increase of thyroid hormone (TH) due to a variety of causes [1] with high metabolic syndrome as the main clinical manifestation. It not only refers to the thyroid but also may involve the whole body, mostly the cardiovascular and nervous systems [2]. Its incidence rate is increasing year by year and becoming younger trend. The 2010 Ten-City Thyroid Disease Epidemiological Survey found that the prevalence of hyperthyroidism was about $1.1 \%$ [3] in young women [4]. TH is one of the essential hormones to maintain normal metabolism of blood lipids and may be involved in the decomposition, synthesis and mobilization of lipids via varied pathways, thereby regulating lipid metabolism. P、TH secretion of patients with hyperthyroidism has increased, which further promotes the intestinal absorption and synthesis of lipids and accelerates its transformation and excretion. It frequently leads to lipid metabolism disorders. This study is to investigate the correlation of blood lipids and their ratios with cystatin $\mathrm{C}$ in patients with hyperthyroidism. It is hereby reported as follows.

\section{General Data and Methods}

General Data. A total of 100 patients with hyperthyroidism visiting our hospital for health examination and our Endocrinology Dept. for treatment from April 2015 to September 2016, who were treated as the study subject. Among the subjects, there were 50 patients with hyperthyroidism: 
21 males and 29 females, aged from 19 to 76 years old with mean age of $(48.62 \pm 13.47)$ years old; 50 patients with subclinical hyperthyroidism: 18 males and 32 females, aged from 23 to 79 years old with mean age of $(46.37 \pm 12.76)$ years old; and 50 healthy subjects taking health examination at the same period, were included as the control group: 25 males and 25 females aged from 20 to 81 years with mean age of $(45.76 \pm 13.26)$ years old. There was no significant difference in sex and age among the three groups $\left(\chi^{2}=2.896, \mathrm{P}=0.602 ; \chi^{2}=0.809, \mathrm{P}=0.586\right)$. The followings were excluded: with a history of liver and kidney diseases, hypertension and coronary heart disease (CHD), dyslipidemia of other causes. And no medication that may impact thyroid functions and blood lipids were taken in the recent three months.

Methods. Detection methods All subjects were fasting for more than $12 \mathrm{~h}$, who was took $6 \mathrm{ml}$ of venous blood during 7:30 to 9:30 in the morning and dispensed in 2 packs. The serum was separated centrifugally at $4000 \mathrm{r} / \mathrm{min}$ in time. TSH, TT3, TT4, FT3 and FT4 were measured with Gobas E601 Immune Chemiluminescence Apparatus provided by German Roche Limited; and agents were originally provided by Roche.TG, TC, HDL-C and LDL-C were measured with HITACHI-020 Automatic Biochemical Analyzer. TC/HDL-C, TG/HDL-C, LDL-C/HDL-C, LCI = TC $\times$ TG $\times$ LDL-C/HDL-C and non-HDL-C $=$ TC-HDL-C were calculated. CysC was measured with AXSYM Automatic Rapid Immunoassay provided by Abbott USA.

Diagnostic criteria Hyperthyroidism: presence of symptoms or signs; and TSH $<0.27 \mathrm{mIU} / \mathrm{L}$, FT4> $22 \mathrm{pmol} / \mathrm{L}$ and/or FT3> $7.1 \mathrm{pmol} / \mathrm{L}$; subclinical hyperthyroidism: no presence of symptoms or signs; and normal FT3 and FT4, TSH $<0.27 \mathrm{mIU} / \mathrm{L}$.

Statistical Analysis. SPSS 18.0 statistical software was used for data processing. Measurement data was presented in mean \pm standard deviation $(\bar{x} \pm \mathrm{s})$. One-way analysis of variance was used for comparison. Linear correlation analysis was used for the correlation of $\mathrm{TH}$ with blood lipid profile, blood lipid ratios and CysC. $\mathrm{P}<0.05$ denotes significant difference.

\section{Results}

Comparison of Blood Lipids and CysC Levels in each Group. The levels of TC, TG, HDL-C and LDL-C in the hyperthyroidism group were significantly lower than those in the control group ( $\mathrm{P}$ $<0.01)$. There was no significant difference in HDL-C between the subclinical hyperthyroidism and control groups; other than that, its TC, TG, and LDL-C were significantly lower than those in the control group $(\mathrm{P}<0.05)$. Patients with hyperthyroidism and subclinical hyperthyroidism significantly elevated levels of CysC and there was significant difference between the two groups. See Table 1.

Table 1 Comparison of blood lipid and CysC in each group $(\bar{x} \pm \mathrm{s})$

\begin{tabular}{|l|l|l|l|l|l|l|}
\hline Groups & $\mathrm{n}$ & $\mathrm{TC}(\mathrm{mmol} / \mathrm{L})$ & $\mathrm{TG}(\mathrm{mmol} / \mathrm{L})$ & HDL-C $(\mathrm{mmol} / \mathrm{L})$ & LDL-C $(\mathrm{mmol} / \mathrm{L})$ & CysC $(\mathrm{mg} / \mathrm{L})$ \\
\hline Hyperthyroidism & 50 & $3.57 \pm 0.78 *$ & $1.086 \pm 0.59 *$ & $1.128 \pm 0.25 *$ & $1.898 \pm 0.596 *$ & $1.476 \pm 0.384 * *$ \\
\hline $\begin{array}{l}\text { Subclinical } \\
\text { Hyperthyroidism }\end{array}$ & 50 & $4.45 \pm 0.94 *$ & $1.483 \pm 1.135$ & $1.374 \pm 0.287$ & $2.57 \pm 0.778 * *$ & $1.159 \pm 0.146 *$ \\
\hline Controls & 50 & $4.97 \pm 0.96$ & $1.578 \pm 1.200$ & $1.365 \pm 0.326$ & $3.129 \pm 0.380$ & $0.881 \pm 0.176$ \\
\hline
\end{tabular}

Note: Compared with the normal control group, $\quad * \mathrm{P}<0.05, \quad * * \mathrm{P}<0.01$.

Comparison of Blood Lipid Ratios and non-HDL-C in each Group. The levels of TC/HDL-C, TG/HDL-C, LDL-C/HDL-C, LCI, and non-HDL-C in the hyperthyroidism group were significantly lower than those in the control group $(\mathrm{P}<0.05)$;C/HDL-C,TG/HDL-C in the subclinical hyperthyroidism was higher than those in the control group, while LDL-C/HDL-C, LCI, and non-HDL-C significantly decreased, with LCI being the most significant $(\mathrm{P}<0.05)$, followed by LDL-C/HDL-C. The levels of TC/HDL-C, TG/HDL-C, LDL-C/HDL-C, LCI, and non-HDL-C in the hyperthyroidism group were significantly than those in the subclinical hyperthyroidism group $(\mathrm{P}$ $<0.01)$. 
Table 2 Comparison of blood lipid ratios and non-HDL-C in each group ( $\bar{x} \pm \mathrm{s}$ )

\begin{tabular}{|l|l|l|l|l|l|l|}
\hline Groups & $\mathrm{n}$ & TC/HDL-C & TG/HDL-C & LDL-C/HDL-C & LCI & non-HDL-C \\
\hline Hyperthyroidism & 50 & $\begin{array}{l}3.570 \pm 0.78 \\
*\end{array}$ & $\begin{array}{l}1.086 \pm 0.59 \\
*\end{array}$ & $1.23 \pm 0.254 *$ & $\begin{array}{l}1.898 \pm 0.596 * \\
*\end{array}$ & $\begin{array}{l}2.317 \pm 0.77 \\
*\end{array}$ \\
\hline $\begin{array}{l}\text { Subclinical } \\
\text { Hyperthyroidism }\end{array}$ & 50 & $\begin{array}{l}4.452 \pm 0.94 \\
*\end{array}$ & $1.483 \pm 1.14$ & $1.38 \pm 0.287 *$ & $\begin{array}{l}2.574 \pm 0.778 * \\
*\end{array}$ & $\begin{array}{l}3.087 \pm 0.87 \\
*\end{array}$ \\
\hline Controls & 50 & $3.804 \pm 1.017$ & $1.320 \pm 1.314$ & $2.412 \pm 0.824$ & $23.434 \pm 29.408$ & $3.600 \pm 0.935$ \\
\hline
\end{tabular}

Note: Compared with the normal control group, $\quad * \mathrm{P}<0.05, \quad * * \mathrm{P}<0.01$.

Correlation Analysis among Thyroid Hormones and Blood Lipid Profile, its Ratios, non-HDL-C and CysC. TSH was positively correlated with TC, TG, HDL-C, LDL-C, TC/HDL-C, TG/HDL-C, LDL-C/HDL-C, LCI and non-HDL-C. In addition to $\mathrm{P}=0.007$ for TG, $\mathrm{P}<0.001$ for the remaining. FT3, FT4, T3 and T4 were negatively correlated with TC, TG, HDL-C, LDL-C, TC/HDL-C, TG/HDL-C, LDL-C/HDL-C, LCI and non-HDL-C. In addition to $\mathrm{P}=0.03$ and 0.007 for TG/HDL-C with T3 and T4, respectively, $\mathrm{P}<0.001$ for the remaining. Comprehensive analysis showed that as to the correlation of TH with blood lipid ratio, non-HDL-C and CysC, TC showed the highest correlation followed by non-HDL-C, LDL-C and LCI.

\section{Discussions}

TH can accelerate the mobilization and decomposition of blood lipids and promote the degradation and excretion of cholesterol, leading to the decrease of total cholesterol levels [5]. However, literature showed varied reports regarding the changes of blood lipids in patients with hyperthyroidism. Selim et al [6] reported that the decreases of TC and LDL-C and FT3 and FT4 of patients with hyperthyroidism were significantly negatively correlated with TC, LDL-C and HDL-C and significantly positively correlated with TG; TSH was significantly correlated with TC, LDL-C and HDL-C and significantly negatively correlated with TG [7]. Xiaoqing et al [8] reported that TC, TG, HDL-C and LDL-C's levels of patients with hyperthyroidism had significantly lower than those in the control group; after treatment, the significantly decrease of FT3 and FT4 and TSH, TC, TG, HDL-C, and LDL-C were significantly higher than the levels that before treatment. Sun Jihong et al [9] found that, for patients with hyperthyroidism, the higher the levels of FT3 and FT4, the more significant the decrease of TC, TG, LDL-C and apo E; FT3 was negatively correlated with TC, HDL-C, LDL-C and apo A1; FT4 was negatively correlated with TC, TG, HDL-C, LDL-C and apo A1 with TC being the most significant $(r=-0.498, P=0.000) . ~ G u$ Qing et al [10] reported that patients with hyperthyroidism had higher levels of TC, TG, HDL-C, LDL-C, APOB and LP (a) than those in the control group. The levels of TG, TC, LDL-C and APOB decreased in patients with subclinical hyperthyroidism. The results of this study showed that the levels of TC, TG, HDL-C and LDL-C in patients with hyperthyroidism were significantly lower than those in the control group ( $\mathrm{P}$ $<0.01$ ). In addition to the HDL-C in patients with hyperthyroidism was significant higher than that in the control group ( $\mathrm{P}<0.05), \mathrm{TC}, \mathrm{TG}$ and LDL-C were also lower than those in the control group, which was consistent with the above results. The decrease of TC, TG and LDL-C in patients with hyperthyroidism may be related to the following factors: (1) TH can accelerate the conversion of LDL-C, increase the number and activity of LDL receptors in liver and promote LDL-C clearance, and reduce the level of LDL-C; (2) TH promotes the use of cholesterol in peripheral tissues, accelerates the excretion of bile acids and bile, and reduces blood TC; (3) TH enhances the activity of lipoprotein lipase, accelerates the use of TC in peripheral tissues, and cleanses the clearance rate of TC, which results in decreasing levels of blood TC and TG [11]; (4) TH can promote the reverse transport of cholesterol, accelerate the conversion of cholesterol ester from HDL to LDL, and directly or indirectly reduce TC, TG, HDL-C, and LDL-C. However, it is reported that some patients with hyperthyroidism may have significantly higher TG and LDL-C than those in the control group [12]. Therefore, further in-depth study is required for the changes of blood lipid profile and its mechanism in patients with hyperthyroidism. 
Blood lipid determination is a common indicator for clinical evaluation of the risk and prognosis of CHD. However, not all CHD patients undergo significant changes in all of their lipid-related levels. TC / HDL-C and LDL-C/HDL-C are of more value to CHD diagnosis than a single indicator [13]. TC/HDL-C and LDL-C/HDL-C can accurately reflect the comprehensive level of in vivo lipid metabolism and the risk of CHD. Compared with TC/HDL-C, LDL-C/HDL-C is of more value in predicting carotid atherosclerotic plaque [14]. The results of this study show that TC/HDL-C, TG/HDL-C, LDL-C/HDL-C, LCI, and non-HDL-C in the hyperthyroidism group are significantly higher than those in the control group $(\mathrm{P}<0.01)$; TC/HDL-C and TG/HDL-C in the subclinical hyperthyroidism group are significantly higher those in the control group while LDL-C/HDL-C, LCI, and non-HDL-C are significantly reducing. The decrease of LCI is the most significant followed by LDL-C/HDL-C. It suggests that changes of blood lipid ratios are more significant than single indicator in hyperthyroidism and the calculation of blood lipid ratios may open up new ideas for the prediction of hyperthyroidism complicated with cardiovascular diseases.

Ye et al. [15] suggested that changes in TH levels could cause changes in CysC levels. Xu Yanying [16] reported that serum CysC levels in patients with hyperthyroidism were higher than those in the control group. YU Weiguo et al [15] conducted a Meta-analysis on the relationship between serum $\mathrm{CysC}$ and thyroid functions in 520 patients, finding that patients with hyperthyroidism have significantly elevated $\mathrm{CysC}$ while patients with hypothyroidism had significantly reduced CysC ( $\mathrm{P}<0.001)$. The results of this study showed that the levels of CysC in patients with hyperthyroidism and subclinical hyperthyroidism were significantly higher than those in the control group and there was significant difference between the two groups $(\mathrm{P}<0.05)$. The level of $\mathrm{CysC}$ in hypothyroidism patients was significantly lower than that in the control group which is consistent with the above findings. It suggests that the levels of CysC and thyroid functions closely correlated. However, the mechanism of increasing CysC's levels in patients with hyperthyroidism remains unclear. In vitro studies have shown that $\mathrm{TH}$ stimulates the secretion of CysC [18]. Recent studies have shown that TH may have an effect on CysC [19]. Patients with hyperthyroidism have active basal metabolism, accelerate cell renewal rate/metabolic rate, and promote faster secretion of $\mathrm{CysC}$ in nuclear cells, which may be a direct cause to elevated CysC's levels [20]. However further studies are required to confirm the $\mathrm{CysC}$ levels in patients with hyperthyroidism and consistence of impacts that $\mathrm{TH}$ imposes to all nuclear cells.

Most researchers believed that CysC levels are positively correlated with the severity of coronary artery disease [21]. Jiang Xiaojing et al [22] found that the higher the level of serum CysC, the higher the degree of coronary atherosclerosis degree and the risk of multiple vascular disease and plaque instability. Guan Shikui et al [23] carried out a meta-analysis on the relationship between serum CysC and CHD patients in 977 patients and found CHD patients had significantly higher serum CysC than those in the control group; therefore he believed that high level of serum CysC was closely correlated with CHD. This study found that the serum CysC levels of hyperthyroidism and subclinical hyperthyroidism groups had significantly higher than those in the control group. The level changes and the correlation with CHD have been confirmed. Dynamic monitoring of CysC levels might provide an important basis for the transformation from subclinical hyperthyroidism to hyperthyroidism and the prediction of hyperthyroidism complicated with CHD.

\section{Conclusion}

The study shows that changes of $\mathrm{TH}$ in patients with hyperthyroidism and subclinical hyperthyroidism may impose significant impacts on CysC; the levels of TC/HDL-C, TG/HDL-C, LDL-C/HDL-C, LCI and non-HDL-C in patients with hyperthyroidism are significantly higher than those in the subclinical hyperthyroidism and control groups, which is more significant than the single indicator of blood lipids. The level of $\mathrm{CysC}$ is significantly higher in patients with hyperthyroidism and subclinical hyperthyroidism; and changes in blood lipid ratios of elevated CysC are the risk factors of CHD. Therefore, dynamic monitoring of blood lipid ratios and CysC levels for patients with hyperthyroidism and subclinical hyperthyroidism may contribute to the 
prediction of the transformation from subclinical hyperthyroidism to hyperthyroidism and the prediction of hyperthyroidism complicated with CHD.

\section{Acknowledgement}

This study was supported by a Natural Science Project of Cangzhou Medical College (No.14Z001), a Science and Technology Program Fund Project of Hebei in China (No.15277796D).

\section{References}

[1] F Matano, Y Murai, K Adachi, T Kitamura and A Teramoto. Neurosurg Rev,Vol. 37 (2014) No.2, p.347-356.

[2] MX Li, LM Pan, SC Jian and Y Liu.Journal of Chengdu Medical College,Vol.11 (2016) No.2,p.232-234. (In Chinese)

[3] D Li,W Yang,P Xian,P Liu,X Bao,R Zong and L Duan.Clin Neurol Neurosurg,Vol.115 (2013) No.9, p.1647-1652.

[4] E Ryödi,J Salmi, P Jaatinen, H Huhtala, R Saaristo, M Välimäki,A Auvinen and S Metso Clin Endocrinol (Oxf), Vol. 80 (2014) No.5, p.743-750.

[5] YY Chang and YQ Sun. Chinese Journal of Endemiology,Vol.30(2011)No.5, p.559-562. (In Chinese)

[6] FO Selim and AM Ahmed.Int J Adv Res, Vol. 2(2014) No.1, p.172-181.

[7] Y Wang, HL Ruan, Yi Li, M Zhang and CJ Zhao. Journal of Hainan Medical University, Vol. 22(2016) No.13, p.1418-1420. (In Chinese)

[8] L Xiao, M Guo and RY Fan.Chin J Lab Diagn,Vol. 20 (2016) No.10, p.1679-1681.(In Chinese)

[9] JH Sun, Y Zhang, HR Lu, T Sun, M La ,JP Mu, J Qian and JH Shen. Laboratory Medicine, Vol. 31 (2016) No.7, p.549-552. (In Chinese)

[10] Q Gu, KK Shao and YX Wang.Lab Med Clin,Vol. 11 (2014) No.24, p.3455-3456.(In Chinese)

[11] ZC Yuan and HY Yan.Jiangsu Med,Vol.39 (2013) No.8, p.949-950.(In Chinese)

[12] ZZ Zhang, HM Zhang and XL Fu. Chin J Diffie and Compl Cas,Vol. 15(2016) No.9, p.926-930. (In Chinese)

[13] C Wang and P Zhang.Shandong Medical Journal,Vol.55(2015) No.36, p.99-102. (In Chinese)

[14] X Huang, ZW Cui, W Wu, Y Yang, ZL Cao and H Li. International Journal of Laboratory Medicine,Vol. 36(2015) No.9, p.1274-1276.(In Chinese)

[15] Y Ye, X Gai, H Xie, L Jiao and S Zhang.Endocr Pract,Vol.19(2013) No.3, p.397-403.

[16] YQ Xu. J Med Theor \& Prac, Vol. 28(2015) No.7, p.953-954.(In Chinese)

[17] WG Yu, Z Liu, DF Zhang, L Zhang and YS Gao.Labeled immunoassay and clinic, Vol. 23(2016) No.5, p.579-582,589.(In Chinese)

[18]C Schmid , C Ghirlanda-Keller,C Zwimpfer and E Zoidis, Vol. 419(2012) No.2, p.425-430.

[19] F Wang,W Pan,H Wang,Y Zhou,S Wang and S Pan. Crit Care,Vol. 18(2014) No.1, p.R9.

[20] M Fricker, P Wiesli,M Brändle, B Schwegler and C Schmid.Kidney Int,Vol. 63 (2003) No.5, p.1944-1947.

[21] D Zhang and CG Wang. Med J West China Januar,Vol.26(2014) No.1, p.127-128,131..(In Chinese)

[22] XJ Jiang, XY Chen, H Chen, F Qin, T Liu and Yan YF. Practical Journal of Clinical Medicine, Vol. 13 (2016) No.5, p.99-101.(In Chinese)

[23] SK Guan, Z Liu, FS Zhang, GH Liu, FL Zhang and HJ Li. Labeled immunoassay and clinic, Vol. 22 (2015) No.10, p.994-997.(In Chinese) 